\title{
02; 02.2
}

\section{Масс-спектрометрия молекулы ксилита}

\author{
(C) А.Н. Завилопуло, О.Б. Шпеник, О.В. Пилипчинец
}

Институт электронной фризики НАН Украины, Ужгород, Украина

E-mail: gzavil@gmail.com

Поступило в Редакцию 6 апреля 2017 г.

Описаны методика и результаты масс-спектрометрических исследований выхода положительных ионов, образованных в результате прямой и диссоциативной ионизаций молекул ксилита электронным ударом. В диапазоне массовых чисел $1-170 \mathrm{Da}$ и в интервале энергий бомбардирующих электронов $5-80 \mathrm{eV}$ исследовались масс-спектры молекулы ксилита при разных температурах. Впервые, исходя из энергетических зависимостей эффективных сечений образования ионов при бомбардировке электронным ударом определены энергии появления ионов-фрагментов и исследована динамика ударом, ионов-фрагментов ксилита в интервале температур испарения исходного вещества $340-400 \mathrm{~K}$.

DOI: 10.21883/PJTF.2018.08.45972.16817

Многоатомный спирт ксилит $\left(\mathrm{C}_{5} \mathrm{H}_{12} \mathrm{O}_{5}\right)$, как и этиленгликоль, глицерин, инозит и сорбит, относится к группе полиолов - углеводородных спиртов с общей формулой $R-(\mathrm{OH})_{n}$, где $R$ - углеводородный радикал, а $n$ - число гидроксильных групп, содержащихся в молекуле [1]. Характерной особенностью этих спиртов является то, что гидроксильные группы присоединены к насыщенным атомам углерода. Ксилит - естественный пятиуглеродный сахарозаменитель. Благодаря своим уникальным свойствам и огромному потенциалу биологического воздействия он имеет широкую область применения. Ксилит обладает почти такой же сладостью, как сахароза, при более низкой энергоемкости $(2.4 \mathrm{cal} / \mathrm{g}$ против $4.0 \mathrm{cal} / \mathrm{g})$. Это позволяет использовать ксилит в качестве заменителя сахара в диетических продуктах питания. Отметим, что ксилит является важным промежуточным продуктом углеводного обмена в живых организмах [2]. Кроме того, в щелочной среде он обладает способностью образовывать стабильные металл-ксилитовые комплексы с некоторыми катионами, например $\mathrm{Cu}^{2+}, \mathrm{Ca}^{2+}, \mathrm{Fe}^{2+}[3]$.

Ранее нами были проведены систематические масс-спектрометрические исследования представителей полиолов: молекул глицери- 
на [4] и сорбитола [5], для которых предложены схемы фрагментации, иллюстрирующие наиболее вероятные каналы образования ионовфрагментов при электронном ударе в случае, когда энергия налетающих электронов значительно превышает потенциал ионизации молекулы. В работе [1] показано, что образование оксониевых ионов при фрагментации полиолов может быть следствием локализации заряда на атоме кислорода и протекания $\beta$-разрыва (по отношению к гетероатому) молекулярного иона.

В настоящей работе представлены результаты масс-спектрометрического исследования прямой и диссоциативной ионизации молекулы ксилита электронным ударом в припороговой области энергий. Следует заметить, что в базе данных NIST [6] отсутствуют данные об энергиях ионизации и диссоциации молекулы ксилита. Представленные в [6] массспектры измерены при стандартной энергии ионизации $70 \mathrm{eV}$ в диапазоне масс 26-135 Da, но не указаны температура вещества и условия, при которых эти спектры получены. Поэтому сравнение данных из базы NIST со спектрами масс, полученными в данной работе, возможно только на качественном уровне. Ранее нами было показано [4], что именно температура испарения вещества из источника молекулярного пучка является важным параметром при исследовании процессов фрагментации сложных органических молекул под действием электронов. Выбранный нами наиболее информативный масс-спектрометрический метод изучения процесса диссоциативной ионизации позволяет в одинаковых экспериментальных условиях получить полный масс-спектр исследуемой молекулы, оценить относительный вклад каждого диссоциированного фрагмента и измерить энергетические зависимости сечений прямой и диссоциативной ионизации исследуемых молекул электронным ударом.

Эксперимент выполнен на установке с монопольным масс-спектрометром [4]. Пучок молекул ксилита формировался с помощью многоканального эффузионного источника, который позволял создавать концентрацию исследуемых молекул $10^{10}-10^{11} \mathrm{~cm}^{-3}$ в области взаимодействия с электронами. Калибровка шкалы масс проводилась по изотопам атомов $\mathrm{Ar}$ и Хе, а шкалы энергий - по начальному участку сечения ионизации молекулы $\mathrm{N}_{2}$. Измерения осуществлялись в два этапа: на первом исследовались масс-спектры ксилита при разных энергиях ионизирующих электронов и различных температурах источника молекулярного пучка, а на втором - энергетические зависимости от-

Письма в ЖТФ, 2018, том 44, вып. 8 


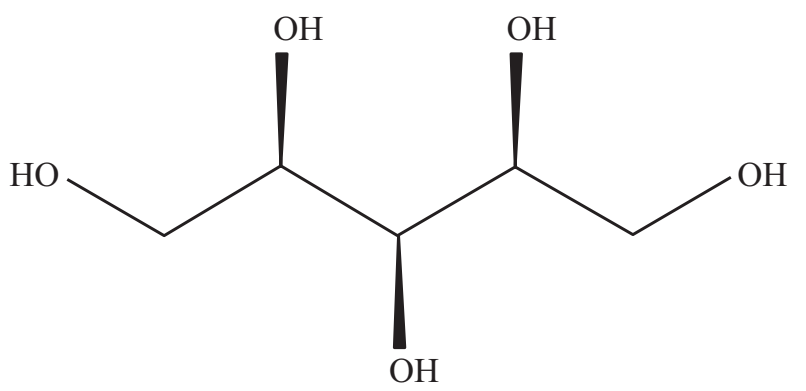

Рис. 1. Структурная формула молекулы ксилита.

носительных сечений диссоциативной и прямой ионизации в диапазоне энергий электронов 5-80 eV.

Как известно [7], при столкновении электрона с многоатомной молекулой возникает нестабильный молекулярный ион $M^{+}$, который, как правило, затем спонтанно распадается на более простой ион и нейтральный остаток (атом, молекула). Наиболее общим направлением фрагментации сложных молекул при электронном ударе является простой разрыв связей углеводородного скелета с образованием ионов оксониевого типа, а также дегидратация молекулярного и фрагментных ионов. Наличие гидроксильной группы увеличивает вероятность диссоциативного распада молекул при ионизации электронным ударом, что обычно приводит к отсутствию в масс-спектре материнского молекулярного пика $M^{+}$[7]. Эта характерная особенность электронной ионизации многоатомных спиртов наблюдалась нами раньше при исследовании моносахаридов: пентозы - глицерин и сорбитол [4,5], гексозы глюкоза и аскорбиновая кислота $[8,9]$.

Рассмотрим процесс взаимодействия электрона с молекулой ксилита. Ионизация молекулы ксилита связана с удалением $n$-электрона, присутствующего в атоме кислорода, поэтому в электронно-невозбужденном состоянии заряд и неспаренный электрон локализованы на атоме кислорода [7]. Такая система является нестабильной, поэтому молекулярный ион $M^{+}$спонтанно распадается на фрагменты.

Структурная формула молекулы ксилита показана на рис. 1. В ней имеются три вершины, связанные с атомом углерода. Если предположить, что первый ион-фрагмент имеет массу $m / z=31\left(\left[\mathrm{CH}_{2} \mathrm{OH}\right]^{+}\right)$, то

Письма в ЖТФ, 2018, том 44, вып. 8 


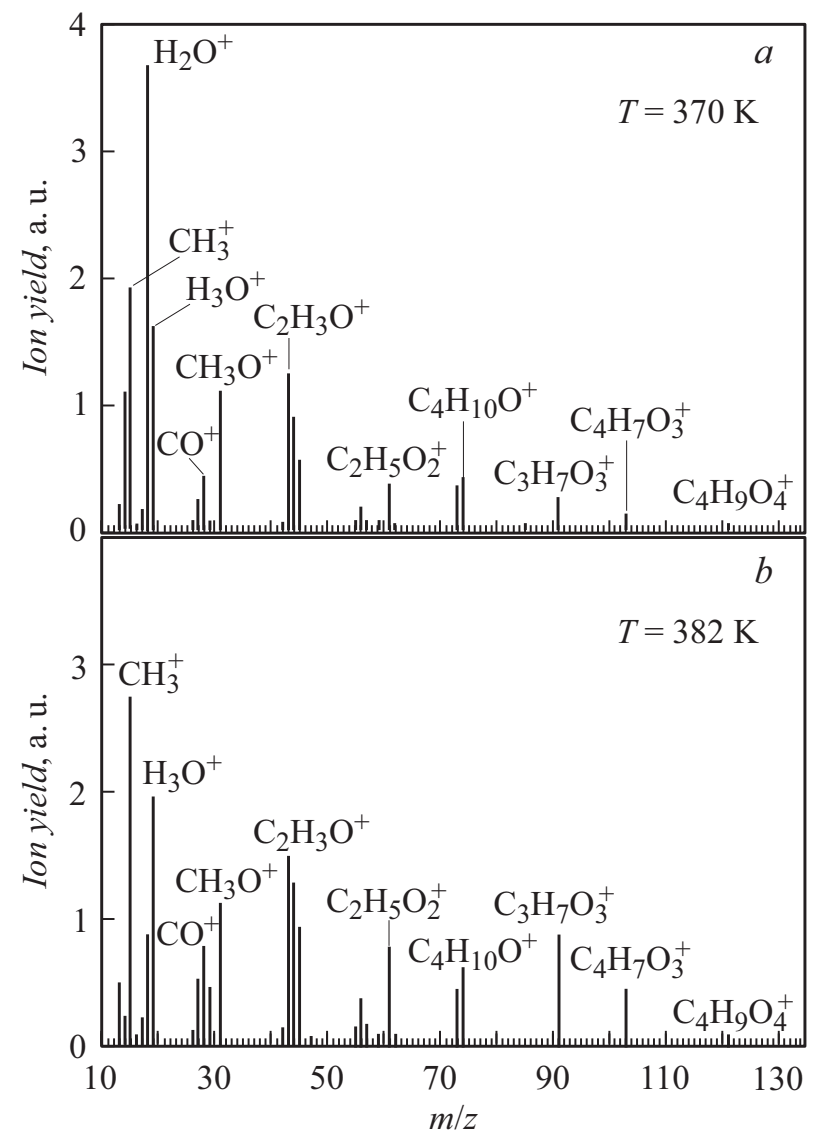

Рис. 2. Масс-спектр молекулы ксилита при различных температурах. $T$, К: $a-370, b-382, c-395$.

остальные осколочные ионы должны появляться с интервалами $30 \mathrm{Da}$ (ион-фрагмент $[\mathrm{CHOH}]^{+}$), т. е в масс-спектре интенсивными будут ионы с $m / z=61\left(\left[\mathrm{CHOHCH}_{2} \mathrm{OH}\right]^{+}\right), m / z=91\left(\left[\mathrm{CHOHCHOHCH}_{2} \mathrm{OH}\right]^{+}\right)$, $m / z=121\left(\left[\mathrm{CHOHCHOHCHOHCH}{ }_{2} \mathrm{OH}\right]^{+}\right)$. Это предположение о формировании ионов-фрагментов с малыми массами подтверждается тем фактом, что, как указывалось выше, молекулярный ион $\mathrm{C}_{5} \mathrm{H}_{12} \mathrm{O}_{5}^{+}$

Письма в ЖТФ, 2018, том 44, вып. 8 


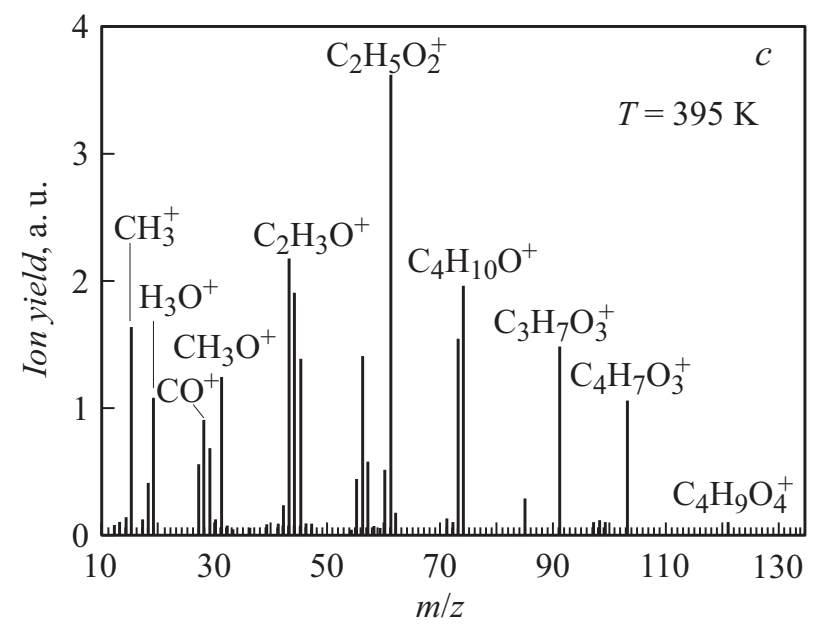

Рис. 2 (продолжение).

$(m / z=152)$ отсутствует в масс-спектре, измеренном нами в интервале $1-170 \mathrm{Da}$.

На рис. 2 показаны масс-спектры молекулы ксилита в диапазоне массовых чисел 10-135 Da, измеренные при разных температурах (энергия ионизирующих электронов $70 \mathrm{eV}$ ). Как видно из рисунка, в соответствии со структурной формулой (рис. 1) общей характеристикой масс-спектров является наличие групп линий, среди которых выделяются масс-пики, соответствующие ионам с $m / z=31,43,61,91,121$ и продуктам вторичной фрагментации осколочных ионов: $\mathrm{CO}^{+}(\mathrm{m} / z=28)$ и $\mathrm{CH}_{3}^{+}(m / z=15)$. Наличие гидроксильных групп ОН в молекуле ксилита приводит к формированию ионов молекулярной воды $\left(\mathrm{H}_{2} \mathrm{O}^{+}\right)$ и гидроксония $\left(\mathrm{H}_{3} \mathrm{O}^{+}\right) \mathrm{c} m / z=18$ и 19 соответственно. Максимальная интенсивность в масс-спектре при $T=395 \mathrm{~K}$ (рис. $2, c$ ) соответствует иону $\mathrm{C}_{2} \mathrm{H}_{5} \mathrm{O}_{2}^{+}(m / z=61)$, который является комплиментарным иону $\mathrm{CH}_{3} \mathrm{O}^{+}(m / z=31)$. Эти ионы образуются при простом разрыве связи $\mathrm{C}-\mathrm{C}$ молекулы ксилита, при этом заряд преимущественно локализован на фрагменте с большей молекулярной массой. Малая вероятность процессов фрагментации, для которых промежуточный однозарядный молекулярный ион распадается на легкий заряженный и тяжелый

7 Письма в ЖТФ, 2018, том 44, вып. 8 


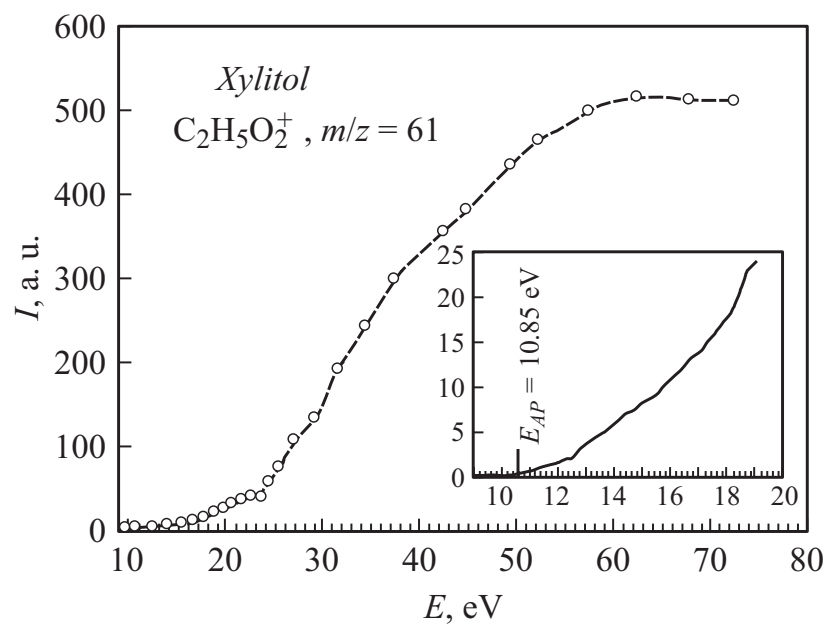

Рис. 3. Энергетическая зависимость сечения образования иона-фрагмента $\mathrm{C}_{2} \mathrm{H}_{5} \mathrm{O}_{2}^{+}$молекулы ксилита. На вставке - начальный участок кривой (шаг по энергии $0.2 \mathrm{eV})$.

незаряженный фрагменты согласно [10], объясняется тем, что эти процессы имеют более высокую пороговую энергию, чем процессы с образованием тяжелого заряженного и легкого нейтрального фрагментов.

Как следует из рис. 2, масс-спектры ксилита провляют существенные различия при изменении температуры от 370 до $395 \mathrm{~K}$. Так, при температуре $370 \mathrm{~K}$, которая близка к температуре плавления ксилита, максимальный пик соответствует молекулярному иону воды (рис. 2,a), с ростом температуры этот пик уменьшается. При $T=382 \mathrm{~K}$ максимальным становится пик, отвечающий иону-фрагменту $\mathrm{CH}_{3}^{+}$(рис. $2, b$ ), а при $T=395 \mathrm{~K}$ максимальным является пик, соответствующий ионуфрагменту $\mathrm{C}_{2} \mathrm{H}_{5} \mathrm{O}_{2}^{+}$(рис. 2,c). С целью выяснения влияния температуры на процесс образования молекулярных ионов-фрагментов нами измерены температурные зависимости для наиболее интенсивных ионных фрагментов в более широком диапазоне $T=340-410 \mathrm{~K}$. Эти зависимости имеют различный вид и особенности с ростом температуры. Для ионов молекулы воды температурная зависимость в начале измеряемого диапазона имеет резкий подъем, а затем слабый линейный

Письма в ЖТФ, 2018, том 44, вып. 8 
Энергии появления $E_{A P}$ ионов-фрагментов и энергия ионизации $E_{I E}$ молекулы ксилита

\begin{tabular}{c|c|c}
\hline Ион & Масса иона, $m / z$ & $\begin{array}{c}\text { Энергия } \\
\text { появления } \\
E_{A P}, \mathrm{eV}\end{array}$ \\
\hline $\mathrm{C}_{2} \mathrm{H}_{5} \mathrm{O}_{2}^{+}$ & 61 & $10.85 \pm 0.25$ \\
$\mathrm{C}_{2} \mathrm{H}_{3} \mathrm{O}^{+}$ & 43 & $11.76 \pm 0.25$ \\
$\mathrm{CH}_{3} \mathrm{O}^{+}$ & 31 & $12.57 \pm 0.25$ \\
$\mathrm{H}_{2} \mathrm{O}^{+}$ & 18 & $13.14 \pm 0.25$ \\
$\mathrm{CH}_{3}^{+}$ & 15 & $12.97 \pm 0.25$ \\
$\mathrm{C}_{5} \mathrm{H}_{12} \mathrm{O}_{5}^{+}$ & 152 & $E_{I E}=10.35 \pm 0.25$
\end{tabular}

рост. Что касается температурных зависимостей ионных фрагментов $\mathrm{CH}_{3}^{+}, \mathrm{CH}_{3} \mathrm{O}^{+}, \mathrm{C}_{2} \mathrm{H}_{3} \mathrm{O}^{+}$и $\mathrm{C}_{2} \mathrm{H}_{5} \mathrm{O}_{2}^{+}$, то для них наблюдается почти плавный рост в области $T=340-370 \mathrm{~K}$, затем динамика меняется и при $T=390 \mathrm{~K}$ начинается резкий рост интенсивности этих линий. Такое поведение температурных зависимостей можно объяснить тем, что процессы образования воды и оксониевых катионов являются конкурирующими [11].

Настроив масс-спектрометр на прохождение ионов определенной массы, мы измеряли энергетические зависимости относительных сечений ионизации и диссоциативной ионизации ионов-фрагментов молекулы ксилита в интервале энергий налетающих электронов 5-80 eV с разным шагом по энергии: в припороговой области $5-20 \mathrm{eV}$ шаг равен $0.2 \mathrm{eV}$, а в области $20-80 \mathrm{eV}-1.0 \mathrm{eV}$. При отключенных потенциалах на отклоняющих электродах масс-спектрометра измерялся полный (суммарный) ток на коллектор ионов, образованных в результате ионизации молекулы ксилита электронами. Таким образом измерена энергетическая зависимость полного относительного сечения образования положительных ионов молекулы ксилита (функция ионизации).

Энергетическая зависимость относительного сечения образования иона $\mathrm{C}_{2} \mathrm{H}_{5} \mathrm{O}_{2}^{+}-$одного из самых интенсивных в масс-спектре (рис. 2,c) - показана на рис. 3, а на вставке приведен пороговый участок кривой, по которому определяется энергия появления $E_{A P}$ ионов. Характерным для данной энергетической зависимости является довольно резкий рост сечения у порога, а также наличие особенностей

7* Письма в ЖТФ, 2018, том 44, вып. 8 
в виде небольших изломов. Интересно заметить, что общий ход этой кривой практически совпадает с ходом энергетической зависимости полного сечения образования положительных ионов молекулы ксилита при ионизации электронным ударом.

Энергетические пороги появления ионов можно определить двумя основными методами: методом фотоионизации и методом ионизации электронным ударом. В основе последнего лежит определение той энергии на энергетической зависимости сечения ионизации, начиная с которой сечение отлично от нуля. Точность определения этой энергии зависит от трех основных факторов: скорости роста сечения в зависимости от энергии бомбардирующих электронов у порога; моноэнергетичности $(\Delta E)$ электронного пучка; точности калибровки шкалы энергий электронов. Следовательно, для точного нахождения порога ионизации к измеренной энергетической зависимости сечения прямой и диссоциативной ионизации необходимо применить определенную процедуру, минимизирующую влияние этих факторов. Нами для определения энергии появления (ионизации) применялась процедура подгонки методом наименьших квадратов с использованием алгоритма Levenberg-Marquardt [12]. По этой методике для всех ионов-фрагментов молекулы ксилита нами определены энергии появления $\left(E_{A P}\right)$. Заметим, что в базе данных NIST [6], так же как и для глицерина [4], эти данные отсутствуют. Как отмечено выше, наиболее интенсивными в масс-спектре являются ионы-фрагменты с $m / z=31,43$ и 61 (рис. 2). Ион $\mathrm{CH}_{3} \mathrm{O}^{+}(m / z=31)$ является характерным для спиртов и всегда присутствует в их масс-спектрах [7]. Образование иона-фрагмента $\mathrm{C}_{2} \mathrm{H}_{3} \mathrm{O}^{+}(m / z=43)$ проходит при элиминировании молекулы воды от иона $\mathrm{C}_{2} \mathrm{H}_{5} \mathrm{O}_{2}^{+}(m / z=61)$. Измеренные нами энергии появления указанных фрагментов, а также потенциал ионизации молекулы ксилита приведены в таблице. Полученные значения величин $E_{A P}$ можно объяснить изменением геометрии и межатомных расстояний в ионизированной молекуле по сравнению с нейтральной, что приводит к существенному снижению энергии связи С-C. Формирование иона-фрагмента $\mathrm{CH}_{3}^{+}$, по-видимому, происходит в процессе вторичной диссоциации, сопровождающейся миграцией протона.

Таким образом, можно заключить, что масс-спектрометрические исследования полиолов дают исчерпывающую информацию об их уникальных свойствах, позволяют оценить степень фрагментации в процессе взаимодействия с электронами, определить параметры меж-

Письма в ЖТФ, 2018, том 44, вып. 8 
молекулярных связей. Впервые по пороговому участку энергетической зависимости эффективного сечения ионизации молекулы ксилита определен потенциал ионизации, а по пороговым участкам относительных сечений диссоциативной ионизации - потенциалы появления наиболее интенсивных в масс-спектре ионов-фрагментов.

\section{Список литературы}

[1] Chen X., Jiang Z.-H., Chen S., Qin W. // Int. J. Biol. Sci. 2010. V. 6. N 7. P. 834-844. doi:10.7150/ijbs.6.834

[2] Mareczky Z., Fehér A., Fehér C., Barta Z., Réczey K. // Period. Polytech. Chem. Eng. 2016. V. 60. N 1. P. 54-59. doi: 10.3311/PPch.8116

[3] Norkus E., Vaiciuniene J., Vuorinen T., Gaidamauskaset E., Reklaitisal J., Jääskeläinen A.S., Crans D.C. // Carbohyd. Res. 2004. V. 339. P. 599-605. doi: 10.1016/j.carres.2003.12.003

[4] Завилопуло А.Н., Шпеник О.Б., Маркуш П.П., Контрош Е.Э. // ЖТФ. 2015. T. 85. B. 7. C. $13-19$.

[5] Chernyshova I., Markush P., Zavilopulo A., Shpenik O. // Eur. Phys. J. D. 2015. V. 69. N 3. P. 80-84. doi: 10.1140/epjd/e2015-50641-7

[6] NIST Standard Reference Database; http:// www.webbook. nist.gov

[7] Лебедев А.T. Масс-спектрометрия в органической химии. М.: БИНОМ, 2003. $493 \mathrm{c}$.

[8] Шиеник О.Б. , Завілопуло А.М. , Агафонова А.С., Романова Л.Г. // Доповіді НАН України. 2008. № 5. С. 96-101.

[9] Завилопуло А.Н., Романова Л.Г., Шиеник О.Б., Агафонова А.С. // ЖТФ. 2009. T. 79. B. 4. C. 19-24.

[10] Афросимов В.В., Басалаев А.А., Березовская Е.А., Панов М.Н., Смирнов О.В., Тулуб А.В. // ЖТФ. 2006. Т. 76. В. 12. С. 16-24.

[11] Nimlos M.R., Blanksby S.J., Qian X., Himmel M.E., Johnson D.K. // J. Phys. Chem. A. 2006. V. 110. N 18. P. 6145-6156. doi: 10.1021/jp060597q

[12] Fiegele T., Hanel G., Torres I., Lezius M., Märk T.D. // J. Phys. B: At. Mol. Opt. Phys. 2000. V. 33. P. 4263-4283; http://iopscience.iop.org/0953-4075/33/20/306

Письма в ЖТФ, 2018, том 44, вып. 8 